Article

\title{
Optimal Insurance Policies in the Presence of Costs
}

\author{
Knut K. Aase
}

Norwegian School of Economics, 5045 Bergen, Norway; Knut.Aase@NHH.NO

Received: 2 June 2017; Accepted: 25 August 2017; Published: 6 September 2017

\begin{abstract}
We reconsider costs in insurance, and suggest a new type of cost function, which we argue is a natural choice when there are relatively small, but frequent, claims. If a fixed cost is incurred each time a claim is made, we obtain a Pareto optimal deductible even if the cost function does not vary with the indemnity. The classical result says that deductibles appear if and only if costs are variable. This implies that when the claims are relatively small, it is not optimal for the insured to be compensated, since the costs outweigh the benefits and a deductible will naturally occur. When we constrain the contract to contain a cap, a non-trivial deductible is Pareto optimal regardless of the assumptions about the cost structure, which is what is known as an XL-contract.
\end{abstract}

Keywords: Pareto optimal risk sharing; administrative costs in insurance; household insurance; reinsurance; $\mathrm{XL}$-contracts

JEL Classification: G00, G22

\section{Introduction}

The paper deals with the impact of the of the insurer's cost structure on optimal, and Pareto optimal, insurance contracts. In particular, it is shown that fixed costs, which are borne by the insurer each time a claim is made, can also explain the existence of deductibles in contracts, just as variable costs, moral hazard or adverse selection do. This result is of special importance when dealing with small, and frequent, losses, such as those observed in everyday non-life insurance. Another result of the paper shows that caps are never Pareto-optimal, and indicates what impact this has regarding the 'optimality', if any, of XL-reinsurance contracts.

As argued below, the result relative to the impact of this type of costs, which we may call quasi-fixed costs for short, is interesting and well understandable. In some way, quasi-fixed costs introduce some variability in the costs by the discontinuity they create. This may explain why the optimal contract has the same structure as the one obtained with continuous, variable costs (see e.g., Arrow 1970; Raviv 1979; Spaeter and Roger 1997).

It seems broadly accepted that deductible policies give the best tradeoff between risk sharing and economizing on costly claim settlements. The presence of insurance costs are often considered as the "best" and most straightforward explanation of deductibles occurring in insurance contracts. There are other explanations, usually involving models of asymmetric information, like moral hazard (Holmstrøm 1979) or adverse selection (Rothschild and Stiglitz 1976). These models are much more complex than simply introducing ex-post costs in the classical model of risk sharing. In these models deductibles appear more or less as a by-product of the analysis. When e.g., moral hazard is present, it is socially optimal that the insured keeps more of the risk than when moral hazard is absent in order to get the incentives right. For example, when the insurer is risk neutral and the classical recipe is that full insurance is Pareto optimal, with moral hazard risk sharing is optimal (second best). When there is adverse selection, the good risks can not be offered full coverage because of the presence of the bad risks. The latter, on the other hand, obtain full insurance (when this is optimal). In both cases 
the insurance customers will end up taking more risk than in the neoclassical case. Whether this risk-sharing takes the form of a deductible, or some other forms of coinsurance is not a central point.

The framework of Pareto optimal risk sharing between an insurer and an insurance buyer is built on Borch's classical theory Borch (1960a, 1960b), and Moffet (1979) was the first to formulate this problem in the neoclassical situation. Deductibles have also been analyzed in the framework of pure demand theory, such as in Arrow (1974), Schlesinger (1981) and Karni (1983). Raviv's analysis of Pareto optimal deductibles in the presence of insurance costs is the classical one (Raviv 1979), and is the first analysis connecting deductibles directly to these costs. For example are some of the results of Arrow clarified through the analysis of Raviv.

Spaeter and Roger (1997) give a unifying treatment by moving the problems to a more general setting, using Gateau derivatives and topology. Gollier (1987) treat various aspects of cost accounting, where he obtains what is termed partially disappearing deductible. His analysis also agrees with one of Raviv's conclusions: there is no deductible when costs are constant.

On one hand, when taking into account sequentially the insurer's problem and the insured's one, neither deductible nor caps are optimal. On the other hand, Pareto optimal contracts contain deductibles almost always when costs are continuous and variable. With this paper, the case of quasi-fixed costs can be added to the list of hypotheses that conduct to deductibles.

In reinsurance excess of loss (XL) contracts are common. This contract contains both a deductible and a cap. This situation can be analyzed just as in the model with an insurance customer and an insurer, where the direct insurer takes the role of the insurance customer, and the reinsurer corresponds to the insurer. We then show that an XL-contract is only constrained Pareto optimal, given that it contains a cap.

The paper is organized as follows: Section 2 develops the setting of the problem, the notation to be subsequently used, and formulates the first order conditions of the optimization problem. In Section 3 we present the main deductible result of the paper. In Section 4 we discuss XL-reinsurance contracts, and in Section 5 we present some relevant background material, containing certain independent results of interest. Section 6 concludes, and proofs are put in two appendices.

\section{Insurance with Costly Claim Settlement}

We start by explaining the cost structure in more detail. The cost function is assumed to be on the following form

$$
C(I)=a \chi_{[I>0]}+c(I)
$$

where $\chi$ is the indicator function of the event $B$,

$$
\chi_{B}= \begin{cases}1, & \text { if } B \\ 0, & \text { otherwise }\end{cases}
$$

Equation (1) means that whenever a claim is made, no matter how small, a cost $a>0$ is incurred, with further costs determined by the function $c(\cdot)$ satisfying the standard conditions: $c(0)=b \geq 0, c^{\prime}(I) \geq 0$, and $c^{\prime \prime}(I) \geq 0$ for all $I \geq 0$. Thus, even if the fixed costs $b=0$, the function $C(\cdot)$ has a discontinuity in $I=0$, with a positive jump size $a$.

Below we show that, if $a>0$, then a non-zero deductible $D>0$ occurs even if $c^{\prime}(I)=0$ for all $I$. This aspect of cost accounting is not captured by the analysis in Raviv (1979), or other related treatments. We claim it to be the important one related to administrative costs in certain lines of insurance.

The motivation for this cost structure is the following. Consider, for example, household insurance. It should be fairly obvious that if all domestic claims are reported, caused by the relatively minor, but frequent accidents that occur in everyday life in the homes of ordinary, insured families, this would be prohibitively expensive for the insurance industry to handle, let alone the mere logistics of the problem. This is where deductibles become important. 
The rest of the model is as follows. The insured faces a random loss $X$ with values $0 \leq x \leq M$, and probability density $f(x)>0$. The indemnity to the insured is $I(x)$ if $X=x$, and the contract has premium $p$. The indemnity function is quite naturally constrained by

$$
0 \leq I(x) \leq x \quad \text { for any } x \geq 0,
$$

implying that $I(0)=0$. Costs of claim settlements are ex post, and given as defined above. The insurer's utility function is $v$, where $v^{\prime}>0$ and $v^{\prime \prime} \leq 0$, and final wealth is $w_{v}-I(x)+p-C(I(x))$ where $w_{v}$ represents initial reserves. The insured's utility function is $u$, where $u^{\prime}>0, u^{\prime \prime}<0$, so the insured is strictly risk averse (otherwise he would not demand insurance). The insured's final wealth is $w_{u}-x+I(x)-p$, where $w_{u}$ is the initial, risk-free part of wealth, and $w_{u}$ and $w_{v}$ are both positive constants.

The premium $p$ in our analysis is simply a positive constant. Presumably it can be expressed as something like

$$
p=(1+\lambda) E^{P}(I(X)+C(I(X))),
$$

but this is not a constraint that we impose on the analysis below. Here $\lambda$ can be thought of as related to a risk premium (and not administrative expenses which are included in $C$ ). This means that the average costs are borne by the consumers, as is the usual situation in all of economics. Alternatively, the expectation operator $E^{P}$ could be exchanged by a $E^{Q}$, where $Q$ is an equivalent risk adjusted probability measure to the given probability measure $P$, so that the risk premium goes via $Q$, in which case $\lambda$ could be set equal to zero (see e.g., Aase (2004) and (2008), who review various aspects of Pareto optimal risk sharing that involve deductibles, and Aase (2002), which is a general review of optimal risk sharing in insurance syndicates).

The variable cost function $c$ is assumed convex in the above. The reason for this assumption, which was also made by Raviv (1979), is that it permits the optimization problem to be convex and the optimal control to lead to a maximum. This simplifies the mathematical problem, while concave costs would not ensure the existence of a maximum.

However, for small and frequent losses (note, we only consider one loss at the time), this assumption is not so intuitive, as some scale economies are expected from the management of small and frequent claims in practice. Actually, it follows from Spaeter and Roger (1997) that the assumption $c^{\prime \prime}>0$ is not needed for the deductible result of this section, $c^{\prime}>0$ is sufficient under some fair assumptions. They also discuss this assumption in detail. Deductibles are optimal when costs are variable and the curvature of the marginal cost only impact the risk sharing between the insurer and the insured beyond the deductible. Since we also say something about this part, we choose to keep the assumption $c^{\prime \prime} \geq 0$.

Pareto optimal contracts $(I, p)$ are generated as solutions of

$$
\max _{I, p} E u\left(w_{u}-X+I(X)-p\right) \text { s.t. } E v\left(w_{v}-I(X)-C(I(X))+p\right) \geq k
$$

As the constant $k$ varies, the Pareto optimal frontier is generated. Since the problem is infinite dimensional, we use Pontryagin's maximum principle (e.g., Seierstad and Sydsæter 1987), where the Hamiltonian of the problem is

$$
\mathcal{H}(I, \lambda)=\left(u\left(w_{u}-x+I(x)-p\right)+\lambda\left(v\left(w_{v}-I(x)-C(I(x))+p\right)-k\right)\right) f(x),
$$

and the Lagrangian is

$$
\mathcal{L}\left(I, \lambda, \mu_{1}(x), \mu_{2}(x)\right)=\mathcal{H}(I, \lambda)+\mu_{1}(x) I(x)+\mu_{2}(x)(x-I(x)) .
$$


If $I^{*}(x)$ denotes the optimal indemnity function, then

$$
\begin{array}{cl}
\mu_{i}(x) \geq 0 & \text { for all } x, i=1,2, \\
\mu_{1}(x)=0 & \text { if } I^{*}(x)>0, \\
\mu_{2}(x)=0 & \text { if } I^{*}(x)<x, \text { and } \\
\mu_{1}(x) I^{*}(x)=0 \text { for all } x, \text { and } \mu_{2}(x)\left(x-I^{*}(x)\right)=0 \text { for all } x .
\end{array}
$$

From this it follows that necessary conditions for a maximum with respect to the indemnity is

$$
u^{\prime}\left(w_{u}-x+I^{*}(x)-p\right)-\lambda v^{\prime}\left(w_{v}-I^{*}(x)-C\left(I^{*}(x)\right)+p\right)\left(1+c^{\prime}\left(I^{*}(x)\right)\right)=0,
$$

for all $x$ such that $0<I^{*}(x) \leq x$, in which case the optimal insurance compensation is given by the differential equation

$$
\frac{d I^{*}(x)}{d x}=\frac{A_{u}\left(W_{u}\right)}{A_{u}\left(W_{u}\right)+A_{v}\left(W_{v}\right)\left(\left(1+c^{\prime}\left(I^{*}\right)\right)+c^{\prime \prime}\left(I^{*}\right) /\left(1+c^{\prime}\left(I^{*}\right)\right)\right.}
$$

where $A_{u}()$ and $A_{v}()$ are the two absolute risk aversion functions, $W_{u}=w_{u}-x+I^{*}(x)-p$ and $W_{v}=w_{v}-I^{*}(x)-C\left(I^{*}(x)\right)+p$. This follows from differentiating the first order condition (5) with respect to $x$, then dividing the result on the first order conditions, and rearranging the final result. Since $0<\frac{d I^{*}(x)}{d x} \leq 1$, by the mean value theorem such policies imply risk sharing between the two parties, i.e., $0<I^{*}(x) \leq x$ for all $x$ such that (5) holds true.

By the maximum principle, the Lagrangian is maximized for the optimal indemnity function, i.e.,

$$
\frac{\partial \mathcal{L}(I, \lambda)}{\partial I}=0
$$

This implies that, when $I^{*}(x)=0$ for $x>0$, this principle gives

$$
J(x):=u^{\prime}\left(w_{u}-x-p\right)-\lambda v^{\prime}\left(w_{v}-b+p\right)\left(1+c^{\prime}(0)\right)+\frac{\mu_{1}(x)}{f(x)}=0,
$$

which means that

$$
J(x):=u^{\prime}\left(w_{u}-x-p\right)-\lambda v^{\prime}\left(w_{v}-b+p\right)\left(1+c^{\prime}(0)\right)=-\frac{\mu_{1}(x)}{f(x)} \leq 0
$$

by the requirement that the Lagrange multiplier function $\mu_{1}(x) \geq 0$.

Similarly, when $I^{*}(x)=x>0$, then the maximum principle gives

$$
K(x):=u^{\prime}\left(w_{u}-p\right)-\lambda v^{\prime}\left(w_{v}-x-a-c(x)+p\right)\left(1+c^{\prime}(x)\right)-\frac{\mu_{2}(x)}{f(x)}=0,
$$

which means that

$$
K(x):=u^{\prime}\left(w_{u}-p\right)-\lambda v^{\prime}\left(w_{v}-x-a-c(x)+p\right)\left(1+c^{\prime}(x)\right)=\frac{\mu_{2}(x)}{f(x)} \geq 0 .
$$

These conditions are also sufficient for a maximum when $u+\lambda v$ is concave in $I$. Due to the discontinuity of $C(I)$ in $I=0, H$ is only concave in $I$ if zero is excluded. However, in the language of Lagrange's method, the "instantaneous kink" at zero is not enough to create a "duality gap" as long as $a$ is not so large that the insurance costs outweigh the benefits of risk sharing. If this is not so, sufficiency of the above conditions are preserved for an inner solution. 
A policy with a deductible is defined as follows. The optimal indemnity function we denote by $I_{D}(x)$. This corresponds to the following policy:

$$
I_{D}(x)= \begin{cases}0, & \text { if } x \leq D ; \\ I^{*}, & \text { if } 0<I^{*} \leq x \text { for } x>D,\end{cases}
$$

where $I^{*}$ is given by the differential Equation (6).

A policy with an upper limit $B$ is defined by Raviv (1979) as follows. The optimal indemnity function we denote by $I_{B}(x)$. This corresponds to the following policy

$$
I_{B}(x)= \begin{cases}x, & \text { if } x \leq B ; \\ I^{*}, & \text { if } 0<I^{*}<x \text { for } x>B,\end{cases}
$$

where $I^{*}$ is given by the differential Equation (6).

The function $J(x)$ is continuous and increasing in $x$, while the function $K(x)$ is continuous and decreasing in $x$, meaning that either (7) or (8) holds, both can not hold for the same $x>0$. If the quantity $L \geq 0$, where $L$ is defined by

$$
L:=u^{\prime}\left(w_{u}-p\right)-\lambda v^{\prime}\left(w_{v}-b+p\right)\left(1+c^{\prime}(0)\right)
$$

then (7) can not hold for any $x>0$, and if $L \leq 0$, then (8) can not hold for any $x>0$. Therefore the optimal solution is one with a deductible, or one with an upper limit. The deductible $D$ and the upper limit $B$ are defined by

$$
u^{\prime}\left(w_{u}-p-D\right)-\lambda v^{\prime}\left(w_{v}-b+p\right)\left(1+c^{\prime}(0)\right)=0
$$

and

$$
u^{\prime}\left(w_{u}-p\right)-\lambda v^{\prime}\left(w_{v}-B-a-c(B)+p\right)\left(1+c^{\prime}(B)\right)=0,
$$

respectively.

If both $D=B=0$, we call the optimal indemnity function $I_{P}(x)$. In the latter case $I_{P}(x)=I^{*}(x)$ for all $x \geq 0$, where $I^{*}$ is determined by the differential Equation (6), with boundary condition $I_{P}(0)=0$.

\section{Pareto Optimal Deductibles in the Presence of Quasi-Fixed Costs}

When there are no ex-post costs, we know (see for example Section 5 below)) that Pareto optimal contracts have no deductibles. From Raviv (1979), Blazenko (1985) and Spaeter and Roger (1997) we know that when there are variable costs $c(I)$, then the Pareto optimal deductible is zero if and only if $c^{\prime}(I)=0$ for all $I$.

In this section, we show that when the cost function is given by (1), then we need to add to this that $a=0$ as well. So for example, when $a>0$ and $c^{\prime}(I)=0$ for all $I$ there is still a non-zero deductible $D>0$. As we have argued in the introduction, this term really captures the essence of cost accounting in certain lines of business, where it is rather intuitive that optimal contracts entail deductibles.

We proceed as follows. First notice that from the relationship (9) we obtain

$$
\frac{d p(D)}{d D}=-\frac{A_{u}\left(w_{u}-p-D\right)}{A_{u}\left(w_{u}-p-D\right)+A_{v}\left(w_{v}+p-b\right)}
$$

which shows that $p(D)$ is a decreasing function of $D$, as expected. The insured's expected utility with deductible $D$ is denoted by $\bar{u}(D)$ and is given by 


$$
\bar{u}(D)=\int_{0}^{D} u\left(w_{u}\right)-x-p(D) f(x) d x+\int_{D}^{M} u\left(w_{u}-x+I_{D}(x)-p(D)\right) f(x) d x,
$$

and the derivative of this with respect to $D$ is

$$
\begin{aligned}
& \frac{d \bar{u}(D)}{d D}=-\frac{d p(D)}{d D} \int_{0}^{D} u^{\prime}\left(w_{u}-x-p(D)\right) f(x) d x \\
& +\int_{D}^{M} u^{\prime}\left(w_{u}-x+I_{D}(x)-p(D)\right)\left(-\frac{d p(D)}{d D}+\frac{\partial I_{D}(x)}{\partial D}\right) f(x) d x .
\end{aligned}
$$

The insurer's expected utility with a deductible is

$$
\begin{aligned}
\bar{v}(D) & =\int_{0}^{D} v\left(w_{v}+p(D)-c(0)\right) f(x) d x \\
& +\int_{D}^{M} v\left(w_{v}-I_{D}(x)+p(D)-C\left(I_{D}(x)\right)\right) f(x) d x,
\end{aligned}
$$

and the derivative with respect to $D$ is

$$
\begin{aligned}
\frac{d \bar{v}(D)}{d D}= & \frac{d p(D)}{d D} \int_{0}^{D} v^{\prime}\left(w_{v}+p(D)-c(0)\right) f(x) d x \\
+ & \int_{D}^{M} v^{\prime}\left(w_{v}-I_{D}(x)+p(D)-a-c\left(I_{D}(x)\right)\right) . \\
& \left(\frac{d p(D)}{d D}-\frac{\partial I_{D}(x)}{\partial D}-c^{\prime}\left(I_{D}(x)\right) \frac{\partial I_{D}(x)}{\partial D}\right) f(x) d x \\
& +v\left(w_{v}+p(D)-c(0)\right) f(D)-v\left(w_{v}+p(D)-a-c(0)\right) f(D) .
\end{aligned}
$$

Provided Pareto optimal contracts contain a non-negative deductible, problem (4) is completed by solving

$$
\max _{D}(\bar{u}(D)+\lambda \bar{v}(D))
$$

We then have the following

Theorem 1. A necessary and sufficient condition for the Pareto optimal deductible $D$ to be equal to zero is $c^{\prime}(I)=0$ for all $I$ and $a=0$.

The proof can be found in Appendix A.

This result tells us that if $a>0$ or $c^{\prime}>0$, there is a non-trivial deductible, and if there are no variable costs so that $c^{\prime}=0$, a deductible still follows provided $a>0$. Thus, with this result, the case of quasi-fixed costs can be added to the list of hypotheses that conduct to deductibles.

\section{Constrained Pareto Optimal Contracts with Caps: XL-Reinsurance Contracts}

As mentioned in the introduction, in insurance practice we often encounter contracts with both a deductible and a cap, or, perhaps, an upper limit and a cap, so one may wonder what role, if any, such contracts may play in optimal contracting.

That Pareto optimal contracts have no cap in the presence of ex-post costs, follows from our above analysis: We know that with ex-post insurance costs, as defined in this paper, Pareto optimal contracts either include a deductible, or an upper limit, not both. By Theorem 1, Pareto optimal contracts turn out to be those with a deductible $D \geq 0$. Caps are simply not a part of Pareto optimal contracts (which also follows from Theorem 5 below).

In our setting upper limit contracts could possibly arise because the natural constraint $I^{*}(x)=x$ for $x>0$ may be violated in the presence of ex-post costs. A contract with a cap, like $I^{*}(x)=F$ for $x \geq x_{F}$ does not violate the natural constraint $0 \leq I^{*}(x) \leq x$, and hence does not enter as a possibility. 
Since caps are parts of real life contracts in the insurance and reinsurance businesses, one may wonder if such contracts satisfy any optimality condition at all. Towards this end, we constrain a contract to contain a non-trivial cap, and ask what effect this may have on the remaining elements of the contract, provided this part satisfy Pareto optimality. The result of this would then be a constrained Pareto optimal contract, constrained to contain a cap.

We first ask if such a contract may possibly contain an upper limit $B>0$. By this we mean a contract of the form

$$
I_{F}(x)= \begin{cases}x & x \leq B \\ I^{*}(x), & \text { if } B<x \leq x_{F} \\ F, & \text { if } x>x_{F},\end{cases}
$$

where $I^{*}(x)$ is given by the differential Equation (6). Here $F \geq 0$ is a constant, and $x_{F}$ is the value of the loss variable where $I^{*}\left(x_{F}\right)=F$. Since the derivative of the Pareto optimal contract $I^{*}$ is less than 1 , it follows that $x_{F}>F$. Let $x_{F}<M$ be some given constant, imposed on the contract.

With reference to Section 5 , Theorem 5 , it turns out that the answer is no. With a constrained cap, the upper limit is $B=0$.

The remaining possibility is that such a contract contains a deductible $D \geq 0$. The contract we consider is then

$$
I_{F}(x)= \begin{cases}0 & x \leq D \\ I^{*}(x), & \text { if } D<x \leq x_{F} \\ F, & \text { if } x>x_{F}\end{cases}
$$

with the same explanation as for the contract above. Such a contract is viable, as we now show.

Theorem 2. A Pareto optimal contract constrained to contain a non-trivial cap, does not contain an upper limit, but consists of a strictly positive deductible $D>0$.

The proof is given in Appendix A.

The result of this theorem is that a (forced) cap alone will imply a non-trivial deductible in the constrained Pareto optimal contract, regardless of assumptions about the cost structure. The result is that an Excess of Loss contract is constrained Pareto optimal in the above meaning.

In addition, a Pareto optimal upper limit does not arise together with a constrained cap.

\section{Related Background Material}

In order to better understand Theorems 1 and 2, the next three subsections contain relevant background material, some of which is known, but some is new material, at least to our knowledge. If we think so, we also provide proofs in the latter case, allocated to Appendix B. We start sequentially with pure demand theory, then move to pure supply theory before we address Pareto optimal policies with costs, where we give a new proof showing why upper limit policies are ruled out.

\subsection{The Theory of Pure Demand}

We start by discussing the much simpler case of pure demand theory. In this field, Arrow (1974) has shown that when the insurance customer's utility function $u$ satisfies $u^{\prime}>0$ and $u^{\prime \prime}<0$, the solution to the problem

$$
\max _{I(x) \geq 0} E u(w-X+I(X)-p) \quad \text { subject to } p=(1+\gamma) E(I(X))
$$

is a contract $I_{D}(x)$ with a deductible $D$, which in this case takes the following simple form: 


$$
I_{D}(x)= \begin{cases}0, & \text { if } x \leq D \\ x-D, & \text { if } x>D\end{cases}
$$

and $D>0$ if and only if the loading $\gamma>0$. Here we may think of the loading as related to a risk premium. Thus, in this framework full insurance is optimal when the premium is actuarially fair only. One way to demonstrate this is to consider non-decreasing contracts $I(x) \geq 0$, and to observe that any deviations from the contract $I_{D}$ satisfying $0 \leq I(x) \leq x$ represent a mean preserving spread in the wealth of the insured, in the sense of Rothschild and Stiglitz (1970). To use this line of proof, it is enough to assume $u^{\prime \prime} \leq 0$.

The impression from results of this type is that contracts with a deductible are somehow "superior". However, and still in the absence of ex-post costs, by also bringing in the supply side, contracts with a deductible can not be Pareto optimal. Even if the premium $p$ is actuarially unfair, the Pareto optimal deductible is zero, and if the insurer is risk neutral, full insurance is Pareto optimal. This follows from the following differential equation for the Pareto optimal indemnity function

$$
\frac{\partial I(x)}{\partial x}=\frac{A_{u}\left(w_{u}-x+I(x)-p\right)}{A_{u}\left(w_{u}-x+I(x)-p\right)+A_{v}\left(w_{v}-I(x)+p\right)},
$$

which, together with the boundary condition $I(0)=0$ yields a unique solution for each $p$. When the premium $p$ varies through a suitable range, this generates the Pareto frontier in $(E u, E v)$-space, where $p$ now takes the role of the Lagrange multiplier $\lambda$ in this regard. Here the functions $A_{u}$ and $A_{v}$ are the absolute risk aversions of the insured and the insurer respectively. From (16) we notice that when $v^{\prime \prime}<0$, then

$$
0<I^{\prime}(x)<1 \quad \text { for all } x \geq 0,
$$

and together with $I(0)=0$ and the mean value theorem, it follows that

$$
0<I(x)<x \quad \text { for all } x>0,
$$

verifying that full insurance is not Pareto optimal when both parties are strictly risk averse. Notice that the natural restriction $0 \leq I(x) \leq x$ is not binding at the optimum for any $x>0$. From this it follows that neither contracts with a deductible, nor contracts with an upper limit are Pareto optimal, since both these contracts would violate the requirement (17) for some $x$. When the insurer is risk neutral, then $I(x)=x$ so full insurance is optimal, regardless of the value of $p$, actuarially fair or not ${ }^{1}$. One would, however, only expect to observe contracts that are also individually rational for both parties, i.e., contracts that are in the core.

These normative conclusions are in agreement with the observation that consumers show a propensity for low or no deductible insurance policies against small to moderate risks. Considering auto insurance as a proxy for insurance against such risks, Pashigian et al. (1966) find that out of a sample, from 1962, of more than 0.8 million insured drivers, 53.8 percent chose the lowest deductible and 45.7 percent chose the next lowest. Cummins and Weisbart (1977) report that a proposal in Pennsylvania to raise the minimum auto insurance deductible from $\$ 50$ to $\$ 100$ during the 1970s was ultimately withdrawn after massive consumer outcry, even though such legislation could have saved consumers millions of dollars each year. Similar attitudes to risk are reported in medical insurance, which is another proxy for moderate risks (the U.S. Bureau of Labor Statistics 1999).

1 If $p \geq(1+\gamma) E I(X)$ is added as a constraint, as in, e.g., Arrow (1970), a deductible will arise, but notice that this then generates only constrained Pareto optimal policies. 


\subsection{The Theory of Pure Supply}

Let us turn to the pure supply-side theory of insurance. Here we consider an insurer with utility function $v$, where $v^{\prime}>0$ and $v^{\prime \prime} \leq 0$, and risk-free reserves $w$, facing the problem

$$
\max _{I(x) \leq x} E v(w-I(X)+p) \quad \text { subject to } p=(1+\gamma) E(I(X))
$$

We can then show

Theorem 3. When the insurer selects to offer insurance contracts $(I(x), p)$, the contract $I^{*}(x)$ solving (18) is one with an upper limit $B: I^{*}(x)=I_{B}(x)$ where

$$
I_{B}(x)= \begin{cases}x, & \text { if } x \leq B \\ B, & \text { if } x>B .\end{cases}
$$

If the loading $\gamma=0$ and $v^{\prime \prime}<0$, then $B=0$.

The proof can be found in Appendix B.

Remark 1. Here the upper limit $B$ is also a cap $(F)$ of the indemnity function. The latter feature is of course important for limiting the insurer's compensation to the insured, which is one reason we studied the XL-contract.

From the last part of the theorem it follows that when $B>0$, necessarily the loading must be strictly positive when the insurer is strictly risk averse.

When the insurer is risk neutral but the premium is unfair, no contract is offered. When the insurer is risk neutral and the premium is fair, the insurer is indifferent between offering full insurance $(B=M)$ and no insurance. When the insurer is risk averse, he requires a premium larger than the actuarially fair one to offer any contract, and if $\gamma$ is sufficiently large a contract is offered, so $B>0$.

For example, and referring to Section 4 where we analyzed the Excess of Loss $(\mathrm{XL})$ contract, recall that these are contracts with a combination of a deductible and a cap. The ceding company takes part of the risk on own account up to a certain value $D$, then cedes the remaining risk to a reinsurer, except that there is some upper bound $F=B$ beyond which the reinsurer is not responsible. The reinsurer only keeps a certain 'layer' of the risk.

We may now go on and find the optimal $B$ given that the contact is one with an upper bound. We limit ourselves to the following:

Theorem 4. In the present framework, the optimal upper bound B $>0$ if and only if $\gamma>0$.

The proof can be found in Appendix B.

The intuition of this result is the following. A risk averse insurer will not offer an insurance contract with an actuarially fair premium, where $B=0$ simply means that the null contract (no insurance offered) is optimal.

The following example illustrates the theory of this section:

Example 1. An insurer with reserves $w=3$ offers insurance against a loss $X$ with probability distribution given in Table 1. Consider a contract with an upper bound $B=1$ and loading $\gamma=0.1$. The insurer's wealth $W_{B}$ is then $\left(2.73 ; \frac{2}{3}, 3.73 ; \frac{1}{3}\right)$. If the insurer instead offers a contract with a deductible $D$ at the same premium as above, then $D=0.5$, and the insurer's wealth $W_{D}$ is instead given by the distribution $\left(2.33 ; \frac{1}{3}, 3.23 ; \frac{1}{3}, 3.73 ; \frac{1}{3}\right)$. It is easy to see that the random wealth $W_{D}$ is a mean preserving spread of $W_{B}$, so all risk averters will prefer to offer the policy with the upper bound B to the one with deductible D (see Rothschild and Stiglitz 1970, or more fundamentally, Blackwell 1951). Here it is seen that $W_{D}=W_{B}+\varepsilon$ in distribution, where the conditional 
distribution of $\varepsilon$ given $W_{B}=2.73$ is $\left(-0.5 ; \frac{1}{2}, 0.5 ; \frac{1}{2}\right)$. Since the insurer prefers the certain outcome 2.73 to the lottery $2.73+\varepsilon$, the fact that $W_{B}$ is preferred to $W_{D}$ actually follows from the substitution axiom.

Table 1. Probability distribution of $X$.

\begin{tabular}{cccc}
\hline $\mathbf{x}$ & $\mathbf{0}$ & $\mathbf{1}$ & $\mathbf{2}$ \\
\hline $\mathrm{P}(\mathrm{X}=\mathrm{x})$ & $\frac{1}{3}$ & $\frac{1}{3}$ & $\frac{1}{3}$ \\
\hline
\end{tabular}

This example also indicates that we could have constructed an alternative proof of Theorem 3 by searching among non-decreasing contracts $I$, and verifying that the associated random wealth $W_{I}$ is a mean preserving spread of $W_{B}$. It may be noted that we have also included variable costs in the above example, and the conclusion still holds.

Consider another example where both the insurance customer and the insurer are in the model:

Example 2. We assume both parties to have negative exponential utility. This means that $u^{\prime}(x)=e^{-a x}$ and $v^{\prime}(x)=e^{-b x}$, where $a$ and $b$ are the absolute risk aversions of the customer and the insurer respectively. The Pareto optimal indemnity function solving Equation (16) is a proportional contract, or a a coinsurance contract of the following form

$$
I(x)=\frac{a}{a+b} x, \text { for } x \geq 0,
$$

valid for any value of the premium $p$. Thus, neither a contract with an upper limit (and a cap), nor one with a deductible is Pareto optimal. The linear contract structure in this example is not so special as it may seem; Pareto optimal indemnity contracts are linear in the loss variable if both parties have HARA utility functions with equal cautiousness parameters.

Imagine that we consider a Pareto optimal contract $(I(x), p)$ that is individually rational for both parties, and assume we can find an upper limit $B>0$ and a loading $\gamma$ such that $p=(1+\gamma) E\left(I_{B}(X)\right)$. Then by Theorem 3 the contract $\left(I_{B}(x), p\right)$ will give the insurer higher expected utility than the Pareto optimal contract. Since the contract with an upper limit is not on the Pareto frontier however, the expected utility of the insurance customer is lower for this contract than for the contract $(I(x), p)$. Thus, there will exist a whole range of contracts on the Pareto frontier that dominate the contract $\left(I_{B}(x), p\right)$, but these will naturally all have higher premiums than $p$.

A similar reasoning can be done for a contract with a deductible $D>0$, but now it is the customer that enjoys a higher expected utility with this kind of contracts. The Pareto dominating contracts in this situation all have lower premiums than $p$. The contract $(I(x), p)$ thus serves as a compromise between these two disjoint sets of Pareto optimal contracts.

It may be observed that a policy with an upper limit in Section 2 does not allow for a cap as in the present section. The reason for this is clear, since the upper limit occurs when costs challenge the natural constraint $0 \leq I^{*}(x) \leq x$. Thus, as we have seen, a Pareto optimal contract in the presence of costs does not contain a cap. This will further be illustrated in the next section.

\subsection{Upper Limit Policies and Insurance Costs}

In the present section we show that in the presence of ex-post insurance costs, Pareto optimal contracts do not contain an upper limit.

In the previous section, we have seen that in the pure theory of insurance supply, and in absence of ex post costs, policies with an upper limit, which is also a cap, have a certain optimality property in that any risk averse insurer prefers to offer such contracts to all other contracts having the same premium. When the insurance customer is also brought into the model, we have seen that Pareto optimal contracts are not of this type, nor does Pareto optimal contracts contain a deductible.

Blazenko (1985) points out that there is an error in Raviv's proof of his Theorem 1, but the result is correct given his assumptions. 
In Theorem 2 of Raviv (1979) the result that Pareto optimal contracts do not contain an upper limit is proved by comparing the slopes of the indifference curves for the insured and the insurer in $(p, B)$-space. In doing so, Raviv employs two different relations for $\frac{d p}{d B}$ depending upon which indifference curve is held constant. While there can be many different connections between $p$ and $B$, there is only one relation for this derivative for any given pair $(p, B)$, derived from Equation (10). To seek a further relationship for this derivative is accordingly inappropriate. Below we present a new proof of this result.

First notice that with Pareto optimal contracts with an upper limit $B$, if they were to exist, $B$ would not serve as a cap on compensations as in (19) of Theorem 3. According to Raviv's definition explained in Section 2, this means that $I_{B}(x):=I(x)=x$ when $x \leq B$, and is given as a solution to the differential equation

$$
\frac{d I(x)}{d x}=\frac{A_{u}\left(W_{u}\right)}{A_{u}\left(W_{u}\right)+A_{v}\left(W_{v}\right)\left(\left(1+c^{\prime}(I)\right)+c^{\prime \prime}(I) /\left(1+c^{\prime}(I)\right)\right.}
$$

when $x>B$, where $W_{u}=w_{u}-x+I_{B}(x)-p$ and $W_{v}=w_{v}-I_{B}(x)-C\left(I_{B}(x)\right)+p$, which follows from differentiating the first order condition (5) with respect to $x$, and then dividing the result by the first order conditions. Since $\frac{d I(x)}{d x}>0$, such policies imply risk sharing for losses above the upper limit $B$.

Next notice that from the relationship (10) we obtain

$$
\frac{d p(B)}{d B}=\frac{A_{v}\left(w_{v}+p-B-a-c(B)\right)\left(1+c^{\prime}(B)\right)+c^{\prime \prime}(B) /\left(1+c^{\prime}(B)\right)}{A_{u}\left(w_{u}-p\right)+A_{v}\left(w_{v}+p-B-a-c(B)\right)}
$$

which shows that $p(B)$ is an increasing function of $B$ under our assumptions on the variable cost function $c$.

The insured's expected utility with an upper limit is denoted by $\tilde{u}(B)$ and is given by

$$
\tilde{u}(B)=\int_{0}^{B} u\left(w_{u}-p(B)\right) f(x) d x+\int_{B}^{M} u\left(w_{u}-x+I_{B}(x)-p(B)\right) f(x) d x,
$$

and the derivative of this with respect to $B$ is

$$
\begin{aligned}
& \frac{d \tilde{u}(B)}{d B}=-\frac{d p(B)}{d B} \int_{0}^{B} u^{\prime}\left(w_{u}-p(B)\right) f(x) d x \\
& +\int_{B}^{M} u^{\prime}\left(w_{u}-x+I_{B}(x)-p(B)\right)\left(-\frac{d p(B)}{d B}+\frac{\partial I_{B}(x)}{\partial B}\right) f(x) d x .
\end{aligned}
$$

The insurer's expected utility with an upper limit is

$$
\begin{aligned}
\tilde{v}(B) & =\int_{0}^{B} v\left(w_{v}-x+p(B)-a-c(x)\right) f(x) d x \\
& +\int_{B}^{M} v\left(w_{v}-I_{B}(x)+p(B)-a-c\left(I_{B}(x)\right)\right) f(x) d x,
\end{aligned}
$$

and the derivative with respect to $B$ is

$$
\begin{aligned}
\frac{d \tilde{v}(B)}{d B}= & \frac{d p(B)}{d B} \int_{0}^{B} v^{\prime}\left(w_{v}-x+p(B)-a-c(x)\right) f(x) d x \\
+ & \int_{B}^{M} v^{\prime}\left(w_{v}-I_{B}(x)+p(B)-a-c\left(I_{C}(x)\right)\right) . \\
& \left(\frac{d p(B)}{d B}-\frac{\partial I_{B}(x)}{\partial B}-c^{\prime}\left(I_{B}(x)\right) \frac{\partial I_{B}(x)}{\partial B}\right) f(x) d x .
\end{aligned}
$$


Provided contracts are of the upper limit type, problem (4) is completed by solving

$$
\max _{B \geq 0}(\tilde{u}(B)+\lambda \tilde{v}(B)) .
$$

We then have the following

Theorem 5. If the variable costs $c$ satisfy $c^{\prime}(I)>0$ with positive probability, then the Pareto optimal contracts are not of the upper limit type. If $c^{\prime}(I)=0$ for all $I$, then $B=0$ is the Pareto optimal upper limit.

The proof can be found in Appendix B.

From the proof we notice that the derivative of the objective function in (24) with respect to $B$ is strictly negative for all $B \geq 0$ under the stated condition, implying that there is no non-negative value of $B$ that satisfies the first order condition. So, under the first condition of the theorem, contracts are not of the upper limit type.

When $c^{\prime}(I)=0$ for all $I$, then $B=0$ solves (24), so the "optimal" upper limit is $B=0$, or in other words, contracts are never of the upper limit type, where the optimal $B^{*}$ satisfies $0<B^{*}<M$.

Intuitively, an increase in $B$ from zero has the effect of increasing insurance coverage for all losses which, in turn, increases the dead-weight loss due to increased insurance costs and therefore is suboptimal.

\section{Conclusions}

When there are no ex-post costs, the pure theory of insurance demand implies that contracts with a deductible are preferred by any risk averse insurance customer among contracts with the same premium. In the pure theory of insurance supply we have demonstrated that any risk averse insurer prefers to offer contracts with a cap and an upper limit to any other contract with the same premium. On the other hand, when Pareto optimality is the criterion, neither of these contract types are optimal.

When there are ex-post costs, it is still the case that policies with an upper limit are not part of the solution. Our main result is that when there are fixed costs triggered whenever a claim is made, deductibles appear in the Pareto optimal policies even if there are no variable costs. With this paper, the case of quasi-fixed costs can be added to the list of hypotheses that conduct to deductibles.

When we constrain the contract to contain a cap, a non-trivial deductible is Pareto optimal regardless of the assumptions about the cost structure.

Acknowledgments: I would like to thank Frøystein Gjesdal for stimulating discussions on cost accounting, and two referees for valuable comments.

Conflicts of Interest: The author declares no conflict of interest.

\section{Appendix A. Proofs of Theorems 1 and 2}

Proof of Theorem 1. The derivative of the objective function in (A4) evaluated at $D=0$ is

$$
\begin{aligned}
& -\frac{d p(0)}{d D} \int_{0}^{M}\left[u^{\prime}\left(w_{u}-x+I_{D}(x)-p\right)\right. \\
& \left.-\lambda v^{\prime}\left(w_{v}-I_{D}(x)+p-a-c\left(I_{D}(x)\right)\right)\right] f(x) d x \\
& +\int_{0}^{M}\left[u^{\prime}\left(w_{u}-x+I_{D}(x)-p\right)\right. \\
& \left.-\lambda v^{\prime}\left(w_{v}-I_{D}(x)+p-a-c\left(I_{D}(x)\right)\right)\left(1+c^{\prime}\left(I_{D}(x)\right)\right)\right] \frac{\partial I_{D}(x)}{\partial D} f(x) d x \\
& +\lambda\left(v\left(w_{v}+p(D)-c(0)\right) f(D)-v\left(w_{v}+p(D)-a-c(0)\right) f(D)\right)
\end{aligned}
$$

which follows from the expressions (A2) and (A3) given in the proof of Theorm 2 below. Here $I_{D}(x)$ is given by (20). If $c^{\prime}(I)>0$ with positive probability, the second term in square brackets is zero from (5). 
The first term in square brackets is then strictly positive with positive probability, and since $\frac{d p(0)}{d D}<0$, the first term is strictly positive. As a consequence, the derivative of the objective function is strictly positive evaluated at $D=0$. Therefore the Pareto optimal deductible is not zero.

When $c^{\prime}(I)=0$ for all $I$ both the first and the second term in square brackets are zero, from (5), and the last term is zero only if $a=0$. Only in this situation the Pareto optimal deductible is zero. If $a>0$ the Pareto optimal deductible is not zero.

When evaluating the derivative of the objective function at any $D$, the additional term to the above expression is

$$
\left.-\frac{d p(D)}{d D} \int_{0}^{D}\left[u^{\prime}\left(w_{u}-x-p\right)-\lambda v^{\prime}\left(w_{v}+p-b\right)\right)\right] f(x) d x
$$

which is less than or equal to zero from (7) provided the cost function satisfies $c^{\prime}(0)=0$. If $c^{\prime}(0)>0$, the sign of this term is ambiguous. Thus, unlike the situation with an upper limit, this derivative may change sign at some $D^{*} \in(0, M)$. If the objective is maximized at $D=M$, the costs of claim settlement overwhelm the advantages of risk sharing.

Proof of Theorem 2. Only the last part is proven here, since the first part is similar to the proof of Theorem 5 given in Appendix B.

The insured's expected utility with deductible $D$ and a cap $F$ is denoted by $\bar{u}_{F}(D)$ and is given by

$$
\begin{gathered}
\bar{u}_{F}(D)=\int_{0}^{D} u\left(w_{u}-x-p_{F}(D)\right) f(x) d x+\int_{D}^{x_{F}} u\left(w_{u}-x+I_{F}^{D}(x)-p_{F}(D)\right) f(x) d x \\
+\int_{x_{F}}^{M} u\left(w_{u}-x+F-p_{F}(D)\right) f(x) d x,
\end{gathered}
$$

and the derivative of this with respect to $D$ is

$$
\begin{aligned}
& \frac{d \bar{u}_{F}(D)}{d D}=-\frac{d p_{F}(D)}{d D} \int_{0}^{D} u^{\prime}\left(w_{u}-x-p_{F}(D)\right) f(x) d x \\
& +\int_{D}^{x_{F}} u^{\prime}\left(w_{u}-x+I_{F}^{D}(x)-p_{F}(D)\right)\left(\frac{\partial I_{F}^{D}(x)}{\partial D}-\frac{d p_{F}(D)}{d D}\right) f(x) d x \\
& +\int_{x_{F}}^{M} u^{\prime}\left(w_{u}-x+F-p_{F}(D)\right)\left(-\frac{d p_{F}(D)}{d D}\right) f(x) d x .
\end{aligned}
$$

The insurer's expected utility with a deductible and a cap is

$$
\begin{aligned}
\bar{v}_{F}(D) & =\int_{0}^{D} v\left(w_{v}+p_{F}(D)-c(0)\right) f(x) d x \\
& +\int_{D}^{x_{F}} v\left(w_{v}-I_{F}^{D}(x)+p_{F}(D)-C\left(I_{F}^{D}(x)\right)\right) f(x) d x \\
& +\int_{x_{F}}^{M} v\left(w_{v}-F+p_{F}(D)-C(F)\right) f(x) d x
\end{aligned}
$$

and the derivative with respect to $D$ is

$$
\begin{aligned}
\frac{d \bar{v}_{F}(D)}{d D}= & \frac{d p_{F}(D)}{d D} \int_{0}^{D} v^{\prime}\left(w_{v}+p_{F}(D)-c(0)\right) f(x) d x \\
+ & \int_{D}^{x_{F}} v^{\prime}\left(w_{v}-I_{F}^{D}(x)+p_{F}(D)-a-c\left(I_{F}^{D}(x)\right)\right) . \\
& \left(\frac{d p_{F}(D)}{d D}-\frac{\partial I_{F}^{D}(x)}{\partial D}-c^{\prime}\left(I_{F}^{D}(x)\right) \frac{\partial I_{F}^{D}(x)}{\partial D}\right) f(x) d x \\
& \int_{x_{F}}^{M} v^{\prime}\left(w_{v}+p_{F}(D)-F-C(F)\right)\left(\frac{d p_{F}(D)}{d D}\right) f(x) d x \\
+ & v\left(w_{v}+p_{F}(D)-c(0)\right) f(D)-v\left(w_{v}+p_{F}(D)-a-c(0)\right) f(D) .
\end{aligned}
$$


Provided constrained Pareto optimal contracts contain a non-negative deductible, the problem is completed by solving

$$
\max _{D \geq 0}\left(\bar{u}_{F}(D)+\lambda \bar{v}_{F}(D)\right) .
$$

The derivative of the objective function with respect to $D$ is

$$
\begin{gathered}
\bar{u}_{F}^{\prime}(D)+\lambda \bar{v}_{F}^{\prime}(D)=-\frac{d p_{F}(D)}{d D} \int_{0}^{D}\left[u^{\prime}\left(w_{u}-x-p_{F}(D)\right)-\right. \\
\left.\lambda v^{\prime}\left(w_{v}+p_{F}(D)-c(0)\right)\right] f(x) d x+\int_{D}^{x_{F}}\left[u^{\prime}\left(w_{u}-x+I_{F}^{D}(x)-p_{F}(D)\right)\right. \\
\left.-\lambda v^{\prime}\left(w_{v}-I_{F}^{D}(x)+p_{F}(D)-C\left(I_{F}^{D}(x)\right)\right)\left(1+c^{\prime}\left(I_{D}(x)\right)\right)\right] \frac{\partial I_{F}^{D}(x)}{\partial D} f(x) d x \\
-\frac{d p_{F}(D)}{d D} \int_{0}^{x_{F}}\left[u^{\prime}\left(w_{u}-x+I_{F}^{D}(x)-p_{F}(D)\right)-\lambda v^{\prime}\left(w_{v}+p_{F}(D)-\right.\right. \\
\left.\left.I_{F}^{D}(x)-C\left(I_{F}^{D}(x)\right)\right)\right] f(x) d x-\frac{d p_{F}(D)}{d D} \int_{x_{F}}^{M}\left[u^{\prime}\left(w_{u}-x+F-p_{F}(D)\right)\right. \\
-\lambda v^{\prime}\left(w_{v}+p_{F}(D)-F-a-c(F)\right] f(x) d x \\
+\lambda\left(v\left(w_{v}+p_{F}(D)-c(0)\right) f(D)-v\left(w_{v}+p_{F}(D)-a-c(0)\right) f(D)\right) .
\end{gathered}
$$

As in the proof of Theorem 4, we notice that if $c^{\prime}(I)>0$ with positive probability, the derivative of the objective function is strictly positive, evaluated at $D=0$, since $\frac{d p_{F}(0)}{d D}<0$. The new term to consider is the effect from the cap via the fourth term on the right-hand side, the integral from $x_{F}$ to $M$; The term in the square brackets in this integral is strictly positive from (5), since the marginal utilities are decreasing and $F<I_{F}^{D}(x)$ for all $x \in\left(x_{F}, M\right)$.

Therefore the constrained Pareto optimal deductible is not zero. When $c^{\prime}(I)=0$ for all $I$, the last two terms are still strictly positive; the last term provided $a>0$, and the next to last term is positive because of decreasing marginal utilities. Thus, we obtain a strictly positive deductible $D$ even if $c^{\prime}(I)=0$ for all $I$ and $a=0$.

\section{Appendix B. Proofs of Theorems 3-5}

Proof of Theorem 3. Since $v^{\prime}>0$, the solution to (18) is the same as the solution to the problem with the inequality constraint $p \leq(1+\gamma) E(I(X))$, because the insurer wants more premiums to less. Using control theory, the Hamiltonian of this latter problem is

$$
\mathcal{H}(I ; \lambda)=v(w-I(x)+p)+\lambda((1+\gamma) I(x)-p)) f(x),
$$

where $\lambda>0$ is a constant, and the Lagrangian is

$$
\mathcal{L}(I ; \mu, \lambda)=\mathcal{H}(I ; \lambda)+\mu(x)(x-I(x)),
$$

where $\mu(x) \geq 0, \mu(x)=0$ if $I^{*}(x)<x$, and $\mu(x)\left(x-I^{*}(x)\right)=0$ for all $x$, where $I^{*}$ denotes the optimal contract. From the maximum principle it follows that the necessary and sufficient first order conditions are found as follows:

$$
\mathcal{H}\left(I^{*} ; \lambda\right) \geq \mathcal{H}(I ; \lambda) \quad \text { for all } I \text { such that } I(x)<x,
$$

which leads to

$$
v^{\prime}\left(w-I^{*}(x)+p\right)=\lambda(1+\gamma) \quad \text { when } I^{*}(x)<x .
$$


Furthermore

$$
\frac{\partial \mathcal{L}\left(I^{*} ; \mu, \lambda\right)}{\partial I}=0 \quad \text { for all } x
$$

which means that

$$
v^{\prime}(w-x+p)-\lambda(1+\gamma)=-\frac{\mu(x)}{f(x)} \leq 0 \quad \text { when } I^{*}(x)=x>0 .
$$

Since the function $v^{\prime}(w-x+p)$ is increasing in $x$, it is clear that there is some $B \geq 0$ for which $v^{\prime}(w-B+p)=\lambda(1+\gamma)$ and (A6) holds true whenever $x \leq B$. From (A5) it is clear that when $x>B$ then $I^{*}(x)=B$, from which the contract (19) follows. $B=0$.

If the insurer is strictly risk averse and the premium is actuarially fair, no contract is offered, or

Proof of Theorem 4. We use the notation

$$
g(B):=E v\left(w-I_{B}(X)+p(B)\right),
$$

where the premium

$$
p(B)=(1+\gamma) E I_{B}(X)=(1+\gamma)\left(\int_{0}^{B} x f(x) d x+B P[X>B]\right),
$$

and $P[C]$ denotes the probability of the event $C$. From this we get that

$$
\frac{\partial p(B)}{\partial B}=(1+\gamma) P[X>B]>0
$$

so the premium $p$ is an increasing function of the upper limit $B$, as expected. Moreover

$$
\begin{gathered}
\frac{\partial g(B)}{\partial B}=P[X>B]\left((1+\gamma) \int_{0}^{M} v^{\prime}\left(w-I_{B}(x)+p(B)\right) f(x) d x\right. \\
\left.-v^{\prime}(w-B+p(B))\right) .
\end{gathered}
$$

The integral can be written

$$
\int_{0}^{B} v^{\prime}(w-x+p(B)) f(x) d x+\int_{B}^{M} v^{\prime}(w-B+p(B)) f(x) d x,
$$

and using the mean value theorem for integrals, it follows that

$$
\int_{0}^{B} v^{\prime}(w-x+p(B)) f(x) d x=v^{\prime}(w-\theta+p(B)) \int_{0}^{B} f(x) d x \text { for some } \theta \in[0, B],
$$

while

$$
\int_{B}^{M} v^{\prime}(w-B+p(B)) f(x) d x=v^{\prime}(w-B+p(B)) P[X>B] .
$$

From this we have

$$
\begin{gathered}
\frac{\partial g(B)}{\partial B}=P[X>B]\left\{( 1 + \gamma ) \left(v^{\prime}(w-\theta+p(B)) P[X \leq B]\right.\right. \\
\left.\left.+v^{\prime}(w-B+p(B)) P[X>B]\right)-v^{\prime}(w-B+p(B))\right\},
\end{gathered}
$$

and setting $B$ equal to zero finally gives 


$$
\left.\frac{\partial g(B)}{\partial B}\right|_{B=0}=P[X>0] \gamma v^{\prime}(w+p(0))>0 \Leftrightarrow \gamma>0,
$$

which proves the theorem.

Proof of Theorem 5. The derivative of the objective function in (24) with respect to $B$ is

$$
\begin{aligned}
& -\frac{d p}{d B} \int_{0}^{B}\left[u^{\prime}\left(w_{u}-p\right)-\lambda v^{\prime}\left(w_{v}-x+p-a-c(x)\right)\right] f(x) d x \\
& +\int_{B}^{M}\left[u^{\prime}\left(w_{u}-x+I(x)-p\right)\right. \\
& -\lambda v^{\prime}\left(w_{v}-I(x)+p-a-c(I(x))\right)\left(1+c^{\prime}(I(x))\right] \frac{\partial I(x)}{\partial B} f(x) d x \\
& -\frac{d p}{d B} \int_{B}^{M}\left[u^{\prime}\left(w_{u}-x+I(x)-p\right)\right. \\
& \left.-\lambda v^{\prime}\left(w_{v}-I(x)+p-a-c(I(x))\right)\right] f(x) d x,
\end{aligned}
$$

which follows from the expressions (22) and (23). Here $I(x)$ is given by (20). If $c^{\prime}(I)>0$ with positive probability, the second term in square brackets is zero from (5). The third term in square brackets is then strictly positive with positive probability, and since $\frac{d p(B)}{d B}>0$, the third term is strictly negative. The first term in square brackets is greater than or equal to zero from (8), so the first term is smaller than or equal to zero. As a consequence, the derivative of the objective function is strictly negative for all $B$. Therefore the Pareto optimal contracts can not be of of the upper limit type when $c^{\prime}(I)>0$ with positive probability.

When $c^{\prime}(I)=0$ for all $I$ both the second and the third term in square brackets are zero for all $B \geq 0$ from (5). The first term is also zero when $B=0$, implying that the Pareto optimal upper limit is $B=0$.

\section{References}

Aase, Knut K. 2008. Optimal Risk-Sharing and Deductibles in Insurance. In Encyclopedia of Quantitative Risk Assessment and Analysis. Edited by E. Melnick and B. Everitt. Chichester: John Wiley \& Sons Ltd., pp. 1230-36.

Aase, Knut K. 2004. Optimal Risk Sharing. In Enclycopedia of Actuarial Science. Edited by J. L. Teugels and B. Sundt. Chichester: Wiley, vol. 2, pp. 676-85.

Aase, Knut K. 2002. Perspectives of Risk Sharing. Scandinavian Actuarial Journal 2: 73-128.

Arrow, Kenneth J. 1970. Essays in the Theory of Risk Bearing. Amsterdam and London: North-Holland.

Arrow, Kenneth J. 1974. Optimal Insurance and Generalized Deductibles. Skandinavisk Aktuarietidsskrift 1: 1-42.

Blackwell, David. 1951. Comparison of experiments. In Proceedings of the Second Berkeley Symposium on Mathematical Statistics and Probability. Berkeley: University of California Press, pp. 93-102.

Blazenko, George. 1985. The Design of an Optimal Insurance Policy: Note. The American Economic Review 75: 253-55.

Borch, Karl Henrik. 1960a. The Safety Loading of Reinsurance Premiums. Skandinavisk Aktuarietidsskrift 1960: $163-84$.

Borch, Karl Henrik. 1960b. Reciprocal Reinsurance Treaties. ASTIN Bulletin 1: 170-91.

Cummins, J. David, and Steven N. Weisbart. 1977. The Impact of Consumer Services on Independent Insurance Agency Performance. Glenmont: IMA Education and Research Foundation.

Gollier, Christian. 1987. Pareto-Optimal Risk Sharing with Fixed Costs per Claim. Scandinavian Actuarial Journal 13: 62-73.

Holmstrøm, Bengt. 1979. Moral Hazard and Observability. Bell Journal of Economics 10: 74-91.

Karni, Edi. 1983. Risk Aversion in the Theory of Health Insurance. In Social Policy Evaluation. Edited by Elhanan Helpman, Assaf Razin and Efraim Sadka. New York: Acdemic Press.

Moffet, Denis. 1979. The Risk Sharing Problem. Geneva Papers on Risk and Insurance 11: 5-13.

Pashigian, B. Peter, Lawrence L. Schkade, and George H. Menefee. 1966. The Selection of an Optimal Deductible for a Given Insurance Policy. The Journal of Business 39: 35-44. 
Raviv, Artur. 1979. The Design of an Optimal Insurance Policy. American Economic Review 69: 84-96.

Rothschild, Michael, and Joseph E. Stiglitz. 1976. Equilibrium in competitive insurance markets. An essay in the economics of imperfect information. Quarterly Journal of Economics 90: 629-50.

Rothschild, Michael, and Joseph E. Stiglitz. 1970. Increasing Risk. I. A Definition. Journal of Economic Theory 2: 225-43.

Spaeter, Sandrine, and Patrick Roger. 1997. The Design of Optimal Insurance Contracts: A Topological Approach. The Geneva Papers on Risk and Insurance Theory 22: 5-19.

Schlesinger, Harris. 1981. The Optimal Level of Deductibles in Insurance Contracts. Journal of Risk and Insurance 48: 465-81.

Seierstad, Atle, and Knut Sydsæter 1987. Optimal Control Theory with Economic Applications. Amsterdam, New York, Oxford and Tokyo: North-Holland.

(c) 2017 by the author. Licensee MDPI, Basel, Switzerland. This article is an open access article distributed under the terms and conditions of the Creative Commons Attribution (CC BY) license (http://creativecommons.org/licenses/by/4.0/). 